\title{
Imaging Cerenkov emission as a quality assurance tool in electron radiotherapy
}

\author{
Yusuf Helo $^{1}$, Ivan Rosenberg ${ }^{2}$, Derek D'Souza ${ }^{2}$, \\ Lindsay MacDonald ${ }^{1}$, Robert Speller ${ }^{1}$, Gary Royle ${ }^{1}$ \\ and Adam Gibson ${ }^{1}$ \\ ${ }^{1}$ Department of Medical Physics and Bioengineering, University College London, \\ London, UK \\ ${ }^{2}$ Department of Radiotherapy Physics, University College London Hospital, London, \\ UK \\ E-mail: Yusuf.helo.10@ucl.ac.uk
}

Received 15 August 2013, revised 28 February 2014

Accepted for publication 28 February 2014

Published DD MMM 2014

\begin{abstract}
A new potential quality assurance (QA) method is explored (including assessment of depth dose, dose linearity, dose rate linearity and beam profile) for clinical electron beams based on imaging Cerenkov light. The potential of using a standard commercial camera to image Cerenkov light generated from electrons in water for fast QA measurement of a clinical electron beam was explored and compared to ionization chamber measurements. The new method was found to be linear with dose and independent of dose rate (to within 3\%). The uncorrected practical range measured in Cerenkov images was found to overestimate the actual value by $3 \mathrm{~mm}$ in the worst case. The field size measurements underestimated the dose at the edges by $5 \%$ without applying any correction factor. Still, the measured field size could be used to monitor relative changes in the beam profile. Finally, the beam-direction profile measurements were independent of the field size within $2 \%$. A simulation was also performed of the deposited energy and of Cerenkov production in water using GEANT4. Monte Carlo simulation was used to predict the measured light distribution around the water phantom, to reproduce Cerenkov images and to find the relation between deposited energy and Cerenkov production. The camera was modelled as a pinhole camera in GEANT4, to attempt to reproduce Cerenkov images. Simulations of the deposited energy and the Cerenkov light production agreed with each other for a pencil beam of electrons, while for a
\end{abstract}


realistic field size, Cerenkov production in the build-up region overestimated the dose by $+8 \%$.

Keywords: Cerenkov light, electron energy, linear accelerator, Monte Carlo, dose

(Some figures may appear in colour only in the online journal)

\section{Introduction}

Cerenkov light is emitted when a charged particle moves in amedium at a velocity exceeding the velocity of light in that medium. The angle of emission and the intensity of the radiation depend on the velocity of the particle and the refractive index of the medium as given by equation (1) (Jelley 1958, Knoll 1988, (Yuan and Wu 1961),

$$
\frac{\mathrm{d} E}{\mathrm{~d} x}=\frac{e^{2}}{c^{2}} \int_{\beta n>1}\left(1-\frac{1}{\beta^{2} n^{2}(w)}\right) w \mathrm{~d} w=\frac{e^{2}}{c^{2}} \int_{\beta n>1} \sin ^{2} \theta w \mathrm{~d} w
$$

where $n$ is the refractive index of the medium, $\beta$ is the ratio of the particle velocity in the medium to the light velocity in the vacuum, $c$ is the speed of light in free space, $e$ is the electron charge and $w$ is the angular frequency of the emitted light. Cerenkov photons are released on a surface of a cone, where the angle of the cone is called the Cerenkov angle, $\theta$ (Jelley 1958, Knoll 1988).

Recently, induced Cerenkov emission from high energy photons or electrons during radiotherapy has been studied in more detail (Axelsson et al 2012, Newman et al 2008). In water, the threshold energy of electrons to induce Cerenkov light is $0.261 \mathrm{MeV}$ assuming $n=1.334$, while in tissue, the threshold energy is $0.213 \mathrm{MeV}$, assuming $n=1.412$ (Tearney et al 1995). Cerenkov radiation occurs across a wide band of the electromagnetic spectrum but absorption in water limits the detectable radiation to the near ultra-violet and visible wavelengths (Jelley 1958, Knoll 1988). Figure 1 shows the theoretical Cerenkov light spectrum with and without the absorption effect of $25 \mathrm{~cm}$ of water.

Electrons interact with matter primarily through Coulomb forces and radiative losses. Coulomb interaction causes excitation and ionization (secondary electrons) in the medium, leading to secondary electrons with an energy spectrum extending from a few $\mathrm{keV}$ to a few MeV. Some of the secondary electrons exceed the Cerenkov production threshold and therefore contribute to the Cerenkov yield. Radiative losses produce Bremsstrahlung radiation which may introduce further ionization which could also emit Cerenkov light (Knoll 1988, Podgorsak 2006).

Different studies show that introducing a variety of radioisotopes (especially $\beta+$ emitters) into an animal produces Cerenkov emissions which can be measured and related to the activity of those isotopes (Robertson et al 2009, Li et al 2010, Hu et al 2010, 2012, Boschi et al 2011), and other studies suggest that measuring Cerenkov light during radiation therapy enables the evaluation of tissue oxygenation (Axelsson et al 2011, Zhang et al 2012). Glaser et al (2013) published a paper about imaging Cerenkov light as a tool for QA in photon therapy. They delivered, a $4 \times 4 \mathrm{~cm}^{2}$ photon beam field with energy equal to $6 \mathrm{MeV}$ to a water phantom and Cerenkov emission was imaged by using a sensitive CCD. Recently, Zhang et al (2013) imaged Cerenkov emission from the surface of flat tissue phantom and compared with the estimated superficial dose deposited by electron beam in that phantom measured by diode. They tested the dose linearity of Cerenkov measurement along with the cross beam profile, 


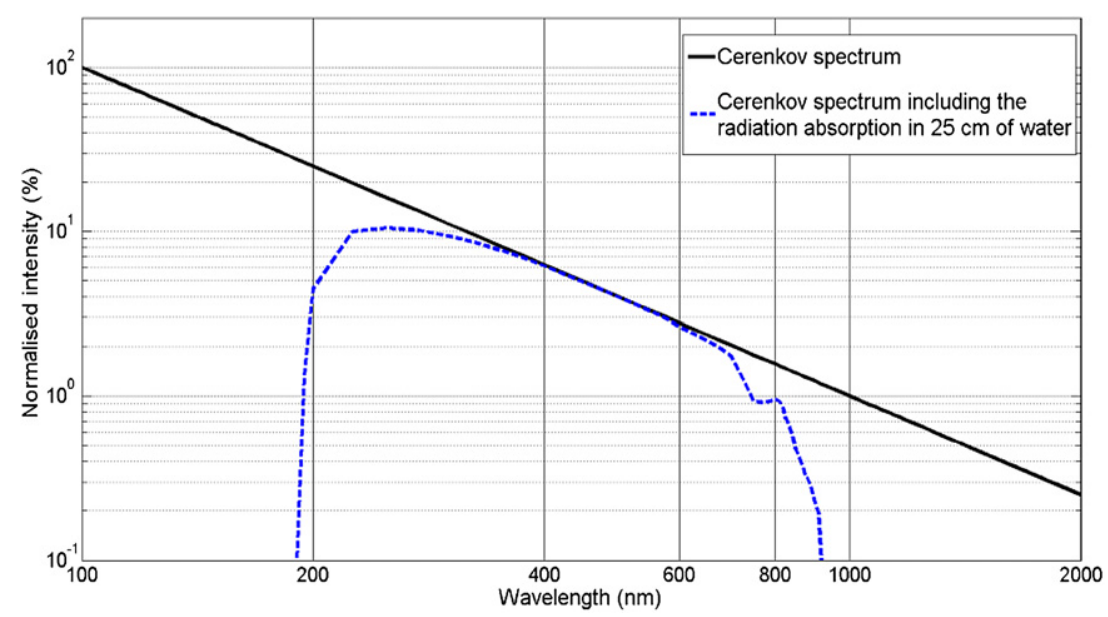

Figure 1. Theoretical Cerenkov light spectrum considering the absorption effect of $25 \mathrm{~cm}$ of water. Drawn using Cerenkov equation and Beer-lambert law in Matlab 7.12.0 (The MathWorks Inc., Natick, MA). Water absorption coefficient was taken from Hale (1973).

while in our study we extend their work to include the dose rate dependence, the field size dependence and the depth profile of Cerenkov images for the first time.

In this work the possibility of imaging Cerenkov emission in electron therapy as a QA tool using a commercial camera is explored. The delivered doses and dose rates are correlated to the measured image intensities in photographs of Cerenkov light. Comparisons are made between the percentage depth dose (PDD) of different electron beam energies with profiles measured from Cerenkov emissions in order to explore whether the latter can be used to check the stability of electron ranges in water. Comparisons are also made between the beam profile of $6 \times 6 \mathrm{~cm}^{2}$ electron beam at $d_{\max }$ with the Cerenkov beam profile at the same depth, to explore whether the latter can be used as a field size verification tool. Imaging Cerenkov light in radiotherapy (electron or photon) is affected, among other things, by: (i) the scattering pattern of electrons inside the water which is energy dependent; (ii) the angular dependence of Cerenkov production, which is also energy dependent; (ii) the refraction of the light when it travels from water to transparent phantom walls then to air. To better understand how these factors influence the expected measurements, a Monte Carlo simulation of the experiment was performed, which incorporated these effects.

\section{Material and methods}

\subsection{Monte Carlo simulation}

The dose deposited and Cerenkov light distribution were investigated using a Monte Carlo simulation [GEANT4.9.6 (Agostinelli et al 2003, Geant4 User's Guide for Application Developers 2012)] of a clinical electron beam with a quasi-Gaussian electron energy spectrum and beam divergence. The simulation was fine-tuned and validated by comparing the calculated electron dose distributions in water against measurements taken with small detectors (NACP parallel plate ionization chambers and diodes) during the commissioning of a Varian linear accelerator (TrueBeam $^{\mathrm{TM}}$, Varian Medical Systems, Palo Alto, CA) at University College London Hospital (UCLH). Electron beams with energies of 6,9 and $12 \mathrm{MeV}$ and field sizes 
of $10 \times 10 \mathrm{~cm}^{2}$ were simulated irradiating a $50 \times 50 \times 50 \mathrm{~cm}^{3}$ water phantom with a wall thickness of $0.5 \mathrm{~cm}$ Perspex.

The Cerenkov light yield was scored between 400 and $720 \mathrm{~nm}$, which corresponds to the sensitivity of commercial cameras. The refractive index and the absorption length of water were added with a spectral resolution of $25 \mathrm{~nm}$ (Hale 1973, Kasarova et al 2007). The refractive index of air was assumed to be 1.0 and the absorption length of light in Perspex was assumed to be $1.0 \mathrm{~m}$ at all wavelengths. The refraction and reflection effects as light travels between water, Perspex and air were included in the simulation.

The Nikon D70 SLR digital camera was simulated by approximating it to a pinhole camera. The advantage of simulating a pinhole camera is that it has infinite depth of field, it was not necessary to simulate the complex lens system, there is no vignetting effect, and the resolution of the image is determined solely by the dimension of the hole. The disadvantage, however, is that small aperture means that the collection efficiency is low, so the collected intensity is low unless the integration time is very long. The pinhole was a circle with radius $1 \mathrm{~mm}$, and the image was projected into a sensitive $3 \mathrm{~cm} \times 4 \mathrm{~cm}$ detector pixelated into $0.2 \mathrm{~mm} \times 0.2 \mathrm{~mm}$ pixels. This was chosen as a compromise between acceptable resolution and simulation time.

To simulate the dose and Cerenkov production depth profiles, the deposited energy in the centre of the water phantom and Cerenkov light were scored within a linear array of $5 \times 5 \times 1 \mathrm{~mm}^{3}$ scoring volumes. Cerenkov photons were scored in a particular volume only if they were formed in that pixel; photons travelling through a volume were ignored. The chosen scoring volume size was similar to the size of the ionization chamber used experimentally at UCLH to measure the PDD. All simulations used $5 \times 10^{7}$ electrons unless stated otherwise. In GEANT4, a cut-off value of $0.1 \mathrm{~mm}$ (i.e. approximately $0.1 \mathrm{MeV}$ for electrons in water) was chosen below which the particle is no longer assumed to produce secondary electrons (Agostinelli et al 2003, Geant4 User's Guide for Application Developers 2012).

\subsection{Determination of distribution of light around the phantom}

In order to inform the measurement of the Cerenkov emissions, Monte Carlo simulation was used to predict the light distribution around the phantom. The Cerenkov light distribution around the water phantom was found by scoring the light in the $\mathrm{X}^{-}$and $\mathrm{Y}^{-}$faces just after the Perspex walls. Figure 2 shows the light distribution for a $9 \mathrm{MeV}$ electron beam and the light distribution depth profile as function of angle $\Theta$.

\subsection{Performance tests}

All tests were carried out using a Varian linear accelerator, using electron energies of 6,9 and $12 \mathrm{MeV}$ with source-to-surface distance equal to $100 \mathrm{~cm}$ and a $50 \times 50 \times 50 \mathrm{~cm}^{3}$ water phantom made from $5 \mathrm{~mm}$ thick walls of transparent Perspex (RFA 300, IBA, Belgium). The Cerenkov light was imaged using a CCD camera (Nikon D70, Nikon, Tokyo, Japan) equipped with a standard $50 \mathrm{~mm} \mathrm{f/1.8} \mathrm{Macro} \mathrm{HSM} \mathrm{lens} \mathrm{(Sigma} \mathrm{Corporation,} \mathrm{Kawasaki,} \mathrm{Japan).} \mathrm{The}$ integration time for all images was $30 \mathrm{~s}$, the aperture was f/1.8 and the CCD gain was ISO800. The raw images had a size of $3039 \times 2014$ pixels, and were processed by subtracting a background image that was obtained in the same lighting conditions but with the beam turned off. All images were converted from NEF format which is the raw format of the Nikon camera to Tiff format which retains all the information in the image and can be read into Matlab. All images were tested for saturation and corrected for vignetting. Vignetting is the reduction of an image's brightness at the margin compared to the image centre, which is caused by optical 
(a)

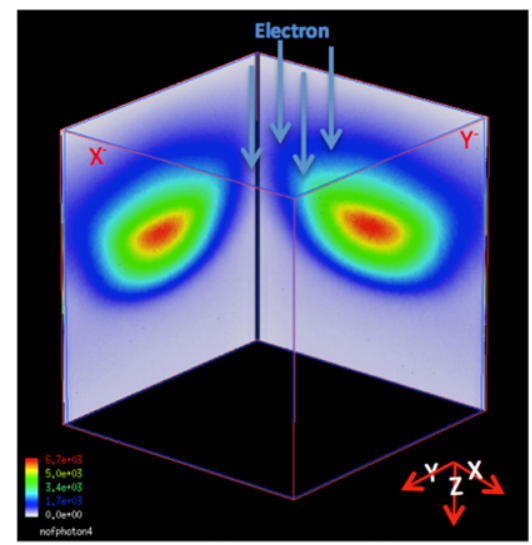

(b)

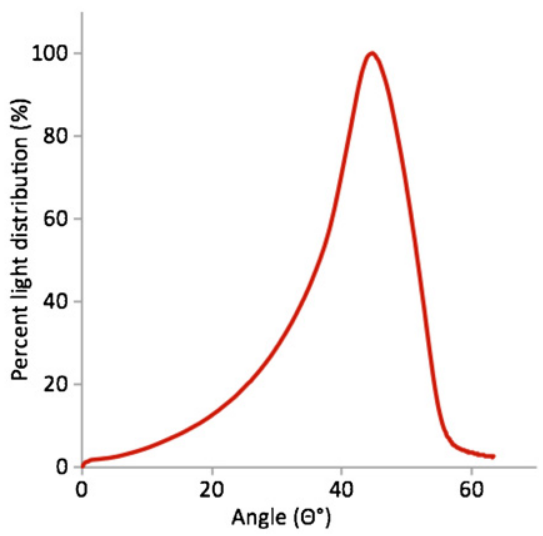

Figure 2. (a) Simulated 2D light distribution in a $50 \times 50 \times 50 \mathrm{~cm}^{3}$ water phantom irradiated by a $10 \times 10 \mathrm{~cm}^{2}$ electron beam with energy equal to $9 \mathrm{MeV}$. Refraction and reflection at boundaries were applied. The scoring area is a mesh pixelated into $0.2 \times 0.2 \mathrm{~cm}^{2}$. (b) Light distribution profile across the $\mathrm{X}^{-}$face as function of angle.

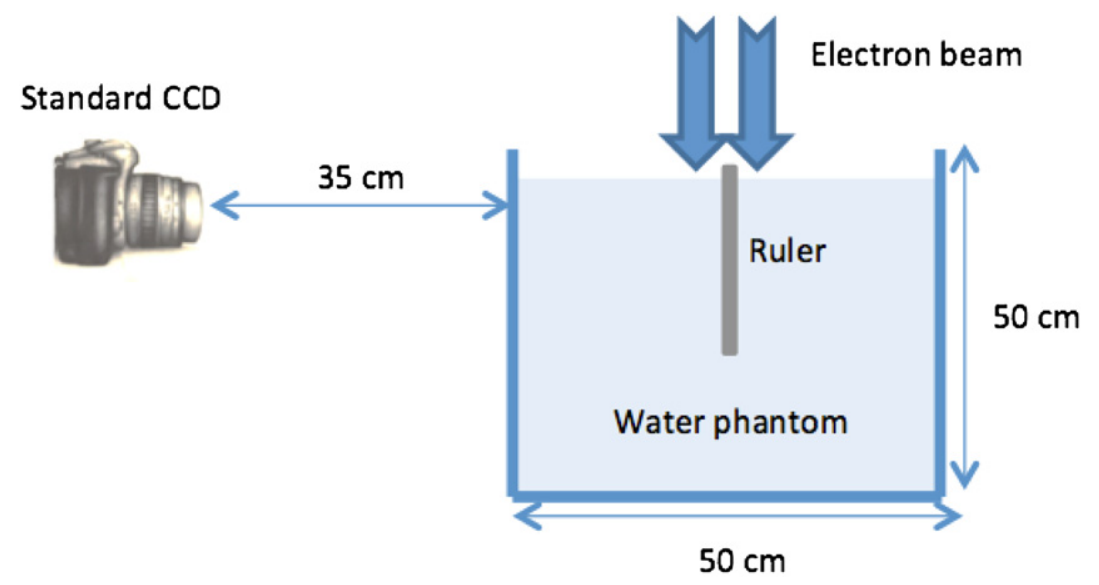

Figure 3. Schematic of the experiment setup.

effects in a multi-lens system (Ray 2002). A vignetting correction factor for each pixel was determined experimentally by imaging a uniformly illuminated field produced by two $50 \mathrm{~Hz}$ white light sources positioned at $45^{\circ}$ projected onto a diffuser sheet. Vignetting correction images were smoothed in Matlab by an averaging filter of 15 pixels diameter to remove the effects of sensor noise. To reduce radiation noise in the images, which predominantly comes from stray x-rays depositing energy on the CCD, and appears as white spots, we applied a $3 \times 3$ pixel median filter (Smith 2003).

The camera was remotely controlled from outside the Linac room by a PC and a $25 \mathrm{~m}$ USB cable. The camera setup is shown in figure 3. All light sources in the room were either turned off or blocked by a black sheet during the experiments. The reproducibility was tested by recording four consecutive images for each measurement and then calculating the standard deviation of each test. The camera was placed at $90^{\circ}$ with respect to the incident electron beam. Distance calibration was performed for each experiment by imaging a metric ruler in the light room conditions placed in the centre of the beam as shown in figure 3 . 


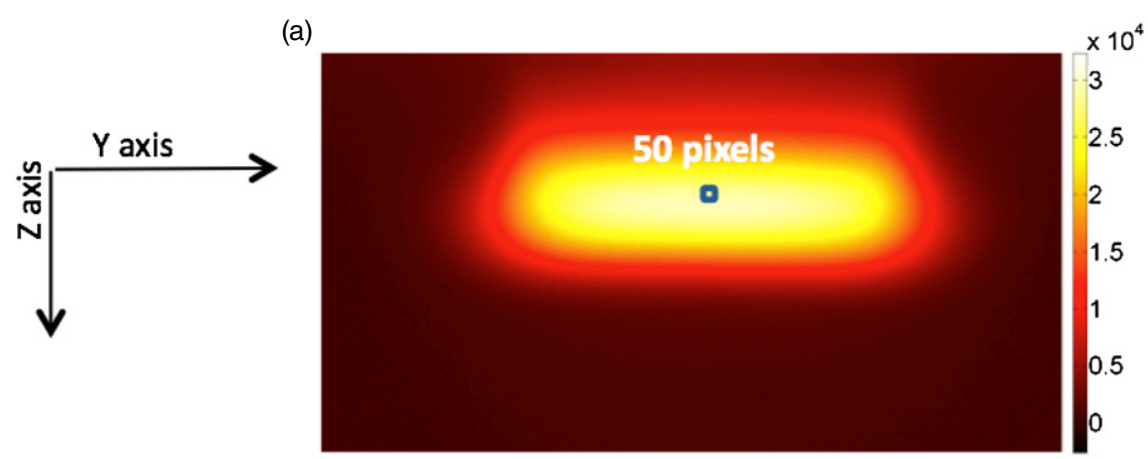

(b)

(c)
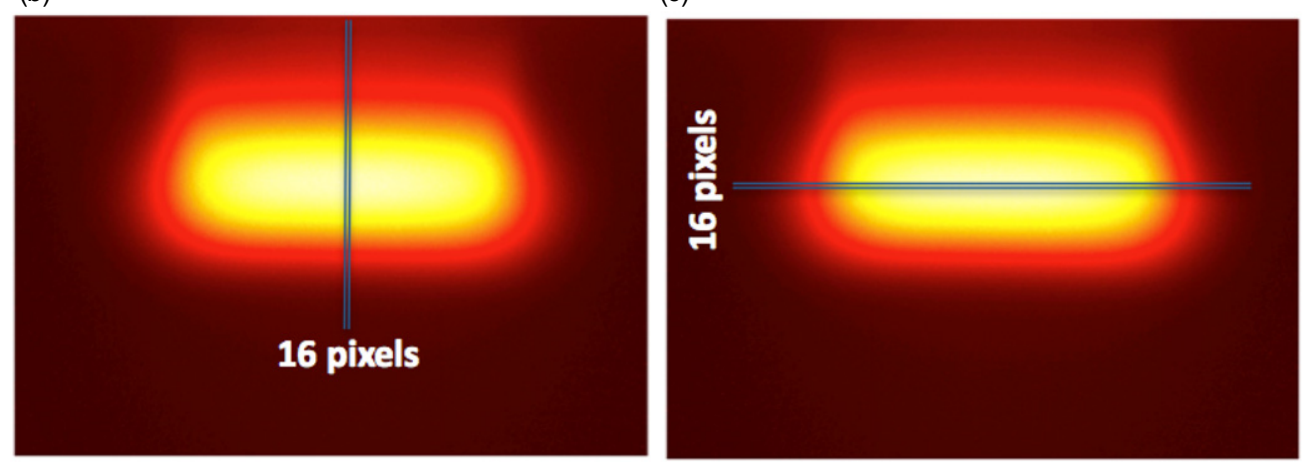

Figure 4. Digital images of Cerenkov light from $12 \mathrm{MeV}$ electron beam showing various regions of integration. (a) The region of interest for linearity test and doserate dependence. (b) The beam-direction profile plane. (c) The transverse profile plane at depth of dose maximum.

\subsection{Dose linearity}

Images were taken with a $10 \times 10 \mathrm{~cm}^{2}$ applicator, at a dose rate of 600 Monitor Units (MU) per minute and $12 \mathrm{MeV}$ electron beam. The dose linearity between 5 and $200 \mathrm{MU}$ was checked by summing the value of a 50 pixel $\times 50$ pixels area as illustrated in figure 4(a). Typical treatment fields deliver dose of about 2 Gy per fraction, corresponding to 200-300 MU, depending on energy and field size.

\subsection{Dose rate dependence}

Measurements were made with $10 \times 10 \mathrm{~cm}^{2}$ applicator delivering $100 \mathrm{MU}$ with a $12 \mathrm{MeV}$ electron beam. The dose rate dependence from 300-900 MU min ${ }^{-1}$ was evaluated by summing the value of 50 pixel $\times 50$ pixels area as illustrated in figure 4(a). The dose rate of a typical treatment is $600 \mathrm{MU} \mathrm{min}^{-1}$.

\subsection{Beam-direction profiles and electron range measurements}

Images were taken with a $10 \times 10 \mathrm{~cm}^{2}$ applicator, $100 \mathrm{MU}$ dose and $600 \mathrm{MU} \mathrm{min}^{-1}$ dose rate. The beam-direction profile relates the change in the intensity values of the image with depth (the $z$-axis in figure 4(b)), averaged over a width of 16 pixels. The practical range of electron beam was evaluated by plotting the beam-direction profiles for 6, 9, and $12 \mathrm{MeV}$ electron beam 
images. The practical electron range is defined as the depth where the tangent to the inflection point of the decreasing portion of the depth-dose curve meets the extrapolated Bremsstrahlung (x-ray) background (Cleland et al 2004). In Cerenkov measurements, we defined the range as the point where the tangent to the inflection point of the decreasing portion of the extrapolated beam-direction profile curve meets the $x$-axis.

\subsection{Field size}

Images were taken at $100 \mathrm{MU}, 600 \mathrm{MU} \min ^{-1}$ dose rate and $12 \mathrm{MeV}$ electron beam for applicator size equal to $6 \times 6 \mathrm{~cm}^{2}$. The transverse profile relates the change in the grey values of the image at a certain depth, orthogonal to the $z$-axis. The transverse profiles at the depth of maximum dose $d_{\max }(2.7 \mathrm{~cm})$ were evaluated by plotting the transverse profile of the field size images as shown in figure 4(c), averaged over a thickness of 16 pixels.

\subsection{Field size dependence}

Measurements were made at $100 \mathrm{MU}, 600 \mathrm{MU} \mathrm{min}^{-1}$ dose rate and $12 \mathrm{MeV}$ electron beam for three different applicator sizes $6 \times 6 \mathrm{~cm}^{2}, 10 \times 10 \mathrm{~cm}^{2}$ and $15 \times 15 \mathrm{~cm}^{2}$. The field size dependence was evaluated by plotting the beam-direction profiles as described in section 2.6 for the different field sizes.

\subsection{Data correction}

Apart from the factors mentioned in the Introduction, the experimental geometry suffered from a couple of distorting effects:

(i) the unequal magnification effects associated with imaging beams with practical field sizes. Cerenkov images are a projection of a 3-D field onto a 2-D plane, which causes image distortion. The geometry and intensity projections depend on the beam field size;(ii) the acceptance angle of the camera, the finite spatial resolution of the camera and the vignetting effect associated with a lens.

The magnification effect can be illustrated by considering a uniform 3D cubic light source in the middle of the water phantom (figure 5(a)). For any plane parallel to the beam direction, the relative intensity of the image can be calculated by:

$$
\frac{I^{\prime}}{I}=\frac{r^{2}}{(r+x)^{2}} .
$$

The relative magnification effect in one dimension can be calculated by:

$$
\frac{y^{\prime}}{y}=\frac{r+x}{r},
$$

where:

$x$ : the distance between a given projection and the midline projection.

$r$ : the distance between midline projection and the projection plane.

$I$ : the intensity of the image of the midline projection.

$I^{\prime}$ : the intensity of the image of the given projection at distance $x$.

$y$ : the width of the midline projection.

$y^{\prime}$ : the width of a given projection.

By summing the contributions of all the projections of light sources for three different cube sizes $(6 \times 6,10 \times 10$ and $15 \times 15)$, and normalizing at the centre of each summed 
(a)
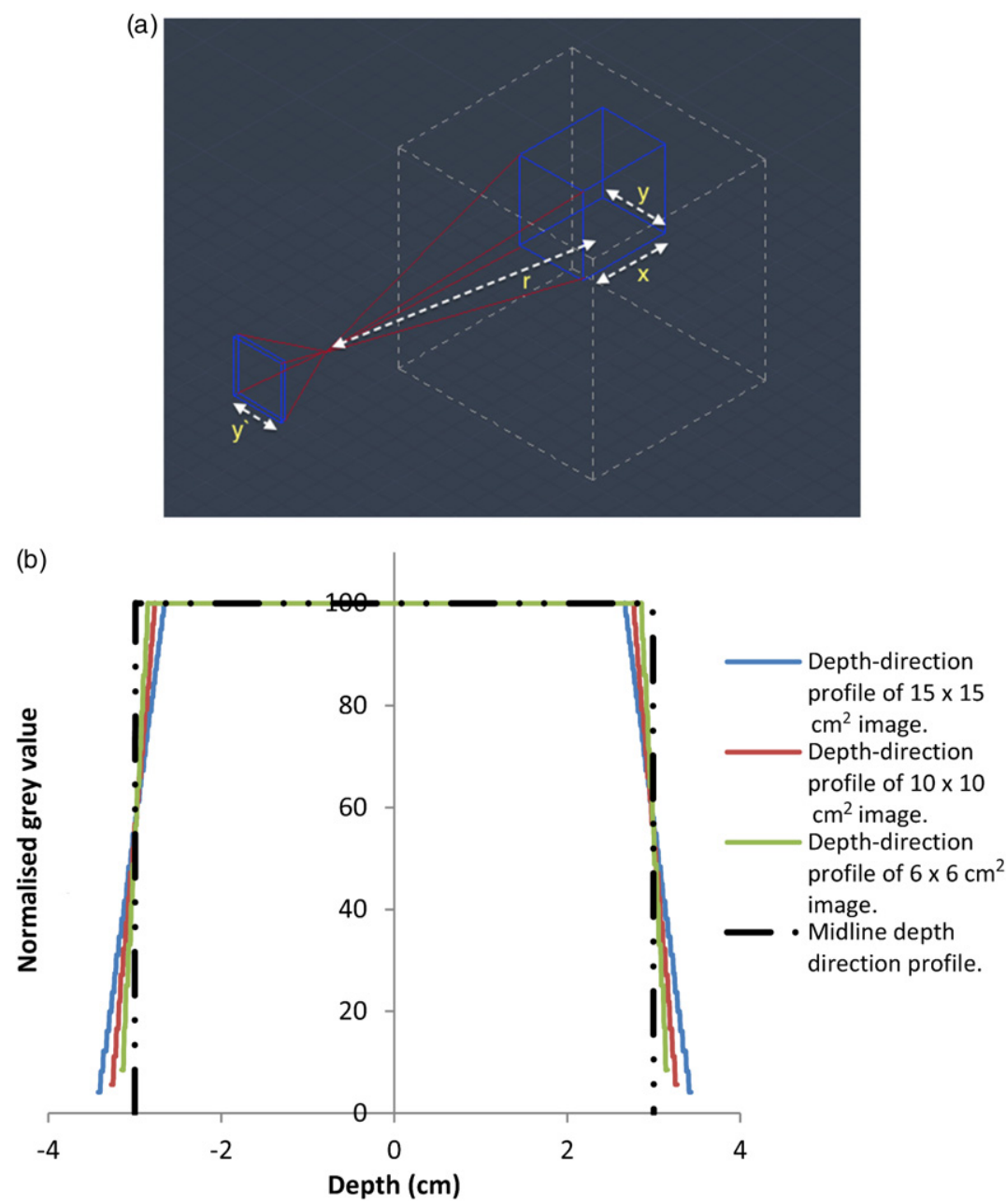

Figure 5. (a) Geometrical magnification and Inverse Law effects on projection of a 3D light cube, as described in the text. (b) Magnification and Inverse Law effects on projection of the $3 \mathrm{D}$ cubic uniform light source described in (a).

projection, one gets the results shown in figure 5(b). This figure shows: (i) the sharp edge of the original light source projects as a slanting penumbra; (ii) the slant of the penumbra is somewhat dependent on the size of the cube and (iii) the full width at half maximum (FWHM) width of the penumbra coincides with the actual width of the light cube. These effects apply both in the beam-direction and in the cross-beam profile. No attempt was made to correct the measured data for this effect; the above analysis should help the understanding of the influence of this effect on the results.

We also investigated the effects of the depth of the field on the spatial resolution and the vignetting problem. We experimentally found that the depth of field associated with our experiment setup reduce the spatial resolution to less than $1 \mathrm{~mm}$ for $10 \times 10 \mathrm{~cm}^{2}$ field size. The vignetting correction factor for each pixel was found experimentally as described in section 2.3. The vignetting correction factor is presented in figure 6. All lenses produce geometrical distortion in the image and for best results these distortions should be corrected. Ideally this should be done by photogrammetry (not tomography) to characterize the mapping function of 


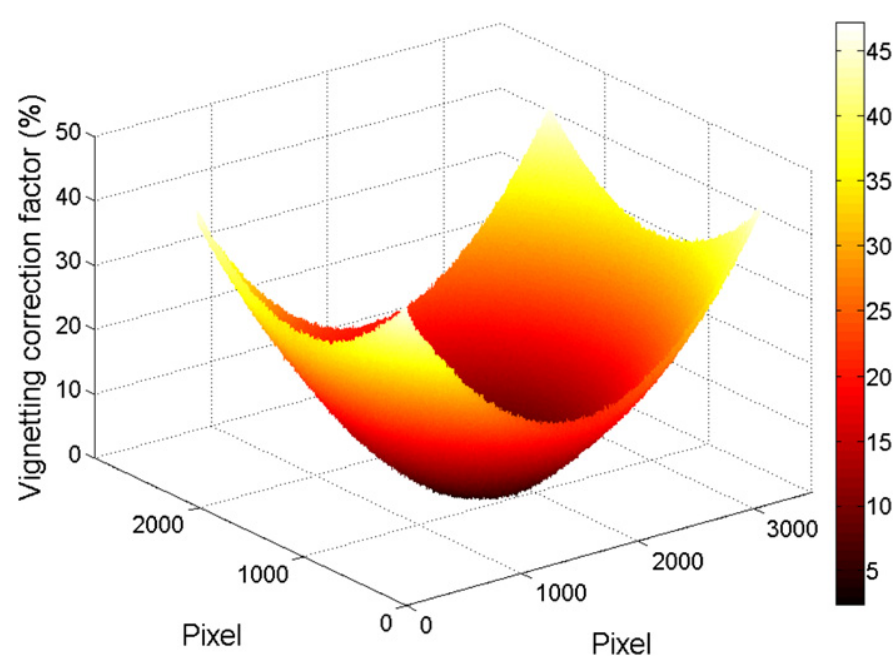

Figure 6. Vignetting correction factor of the used lens as described in 2.3.

the real world onto the image plane with small number (typically seven to nine) parameters (Shortis et al 1998). The vignetting correction factor was applied to all measured images.

\section{Results and discussion}

\subsection{Monte Carlo simulation results}

The maximum in the light distribution detected around the water phantom was found at depths of 24.4 and $24.8 \mathrm{~cm}$ for 6 and $9 \mathrm{MeV}$ electrons beam respectively. This corresponds to $43.6^{\circ}$ and $44.1^{\circ}$ with respect to the incident electron beam for energies of 6 and $9 \mathrm{MeV}$ respectively, which agrees with theoretical predictions. As expected, a more energetic electron beam shifts the maximum intensity peak deeper below the surface of the water.

3.1.1. Mont Carlo simulation input. A quasi-Gaussian energy spectrum, with an additional long tail, and a Gaussian angular distribution were fine-tuned in the simulation to match the simulated depth doses to ionization chamber measurements. The divergence sigma of all beams was $0.6^{\circ}$. The FWHM of the Gaussian energy function was $0.8 \mathrm{MeV}, 1 \mathrm{MeV}$ and 1.2 MeV for $6 \mathrm{MeV}, 9 \mathrm{MeV}$ and $12 \mathrm{MeV}$ electron beams, respectively.

3.1.2. Monte Carlo simulation validation. The depth dose profiles and transverse profiles simulations by Monte Carlo for $6 \mathrm{MeV}, 9 \mathrm{MeV}$ and $12 \mathrm{MeV}$ reproduced data measured with an ionization chamber and diode to within $1 \%$. As an example, the experimental and the simulated PDD and transverse profiles for a $6 \mathrm{MeV}$ electron beam with a $10 \mathrm{~cm} \times 10 \mathrm{~cm}$ applicator are presented in figure 7.

3.1.3. Pinhole simulation. Pinhole camera simulations were used to image Cerenkov light due to 6, 9 and $12 \mathrm{MeV}$ electron beams as illustrated in figure 8, and the corresponding beam-direction profiles extracted from these images are shown in figure 9.

Modelling the camera as a pinhole allowed us to simulate the complex lens-camera combination. We were able to reproduce the experimental Cerenkov images taken in UCLH 
(a)

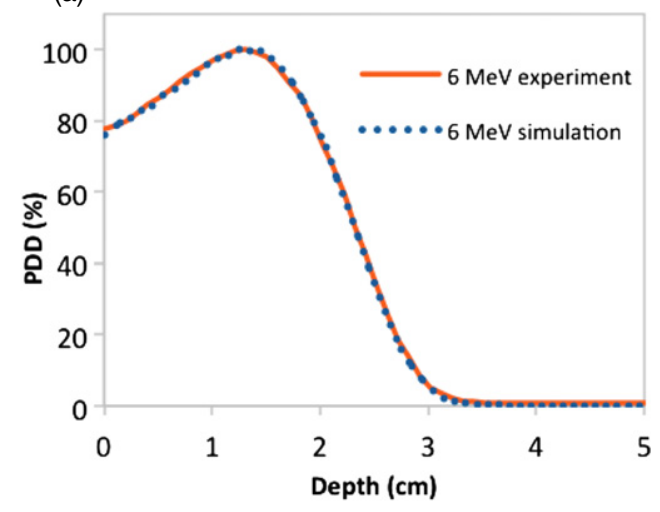

(b)

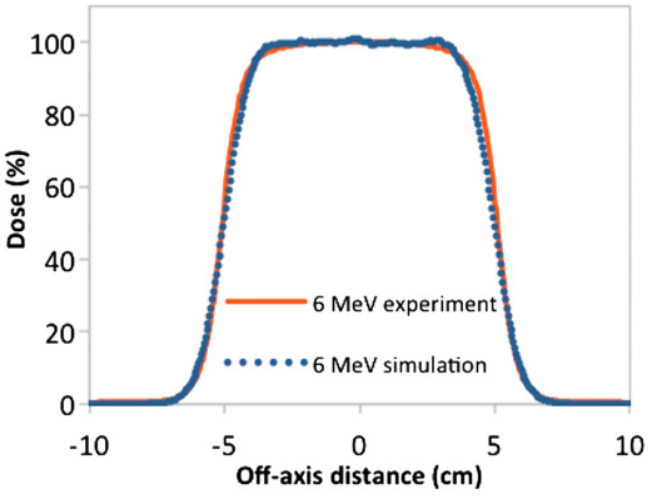

Figure 7. (a) Comparison of depth dose profile between ionization chamber measurement and Monte Carlo simulation, for a $6 \mathrm{MeV}$ electron beam. (b) Comparison of cross-beam profile at depth of maximum dose between diode measurements and Monte Carlo simulation, for a $6 \mathrm{MeV}$ electron beam. The field size was $10 \times 10 \mathrm{~cm}^{2}$.
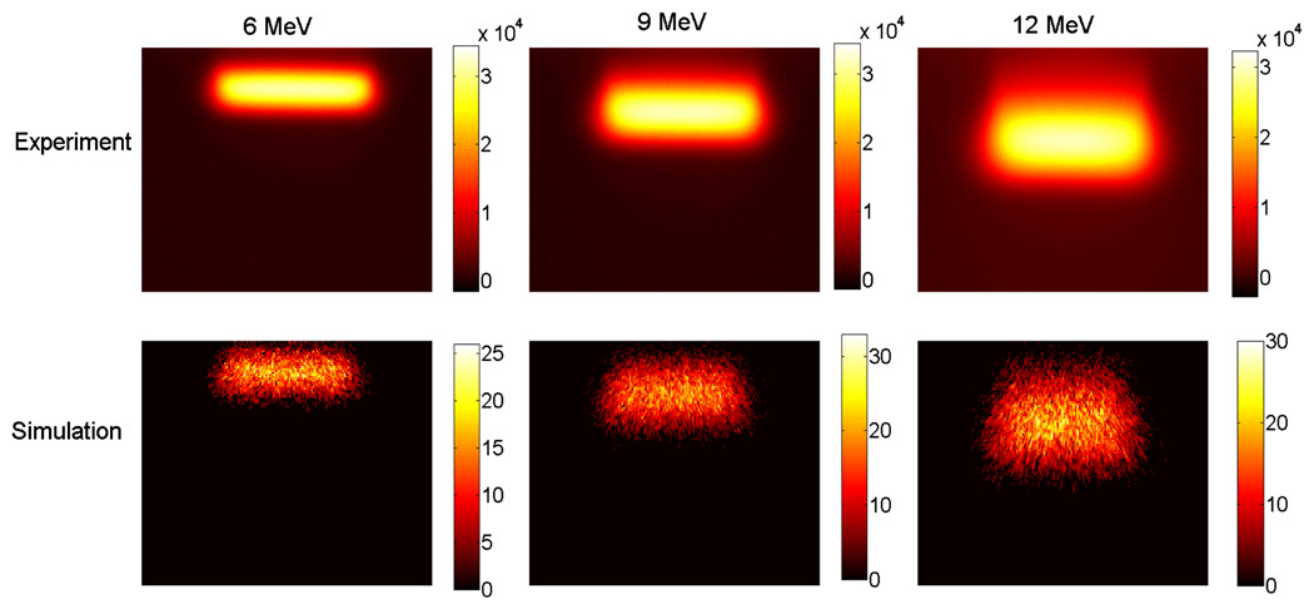

Figure 8. Comparison of experimental Cerenkov images and pinhole camera simulations for 6, 9 and $12 \mathrm{MeV}$ electron beams. Experimental measurements were executed with $10 \times 10 \mathrm{~cm}^{2}$ field size, $200 \mathrm{MU}$ dose and $600 \mathrm{MU} \mathrm{min}^{-1}$ dose rate.

for different energies within $2 \mathrm{~mm}$ accuracy. Note that Cherenkov beam-direction images are very different from the depth doses, especially in the build-up region, due to: (i) the scattering pattern of electrons inside the water which is energy dependent; (ii) the angular dependence of Cerenkov production, which is also energy dependent; (ii) the refraction of the light when it travels from water to transparent phantom walls then to air.

3.1.4. Cerenkov production profile. The relation between the deposited energy and Cerenkov production for $6 \mathrm{MeV}$ pencil electron beam was investigated by scoring the deposited energy and the Cerenkov light which was generated in the same volume. The simulation was repeated for field size $10 \times 10 \mathrm{~cm}^{2}$ as shown in figure 10 .

We found very close relation between the deposited energy per depth and the number of Cerenkov photons produced as a function of depth for the pencil beam of electrons. However, 


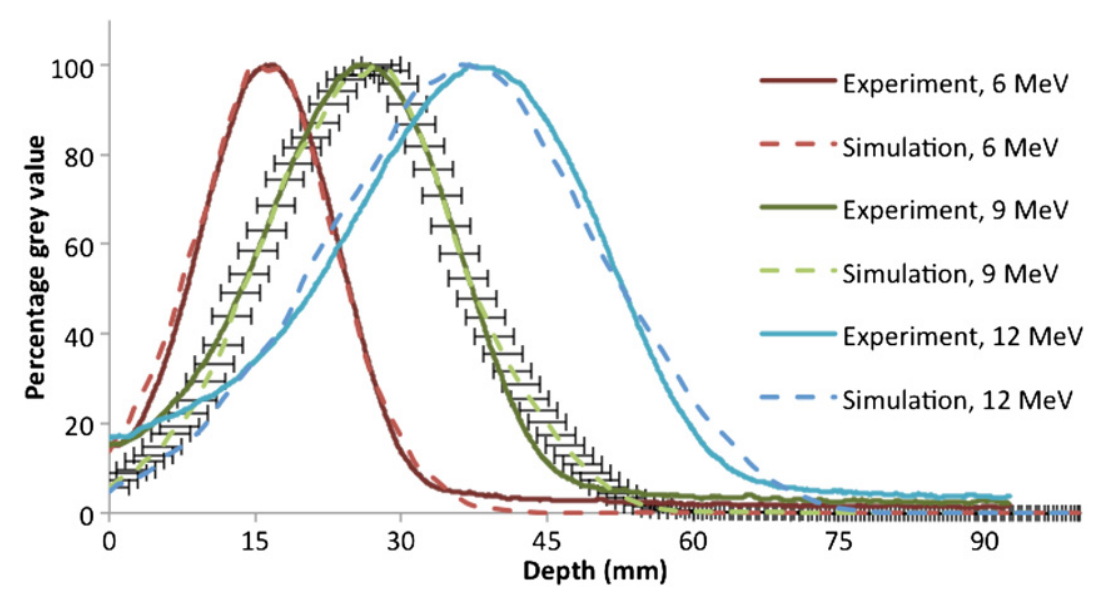

Figure 9. Beam-direction profiles for experimental and simulation Cerenkov images for a $10 \mathrm{~cm} \times 10 \mathrm{~cm}$ field size. The error bars are due to the diameter of the pinhole and the pixel size, and are illustrated just for $9 \mathrm{MeV}$ electron beam.

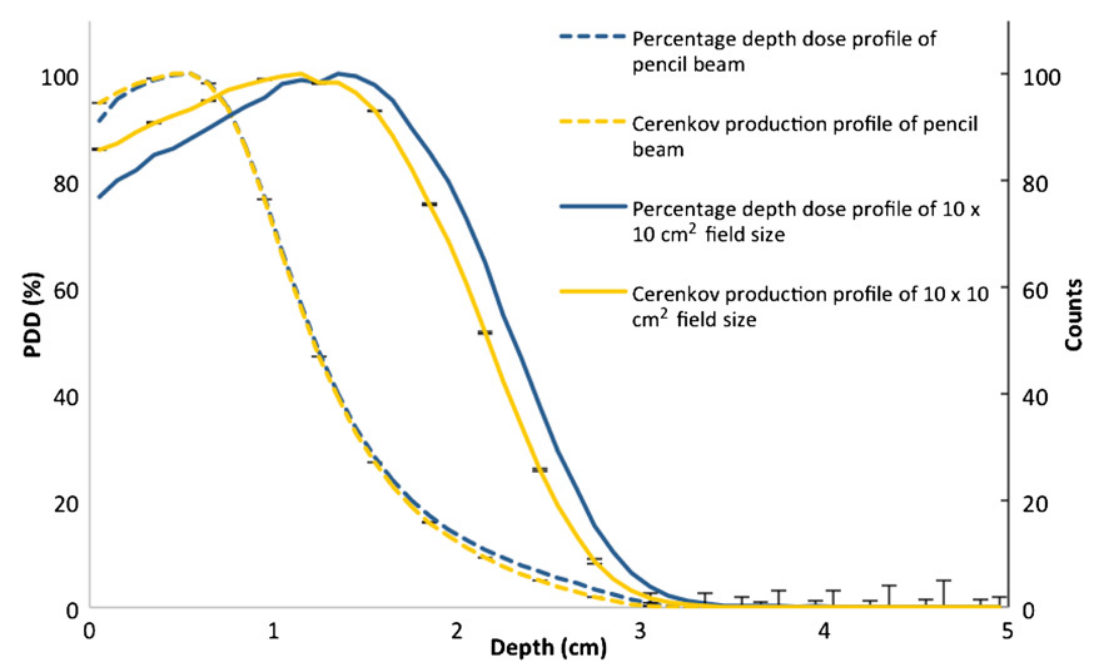

Figure 10. Comparison between simulated energy deposited profile and simulated Cerenkov production profile for two $6 \mathrm{MeV}$ electron beams, one a pencil beam and the other with a field size of $10 \times 10 \mathrm{~cm}^{2}$. The scoring volume is a $0.5 \times 0.5 \mathrm{~cm}^{2}$ square with step equal to $0.1 \mathrm{~cm}$. Error-bars illustrate the typical statistical standard deviation of the simulation data for Cerenkov production profile.

with a $10 \times 10 \mathrm{~cm}^{2}$ field size, the Cerenkov production profile tended to overestimate the dose at the build-up region and it reaches a maximum at a different depth than the depth dose curve. This could be due to the simplified electron energy and divergence model used (section 3.1.1) compared to the real distribution from the applicator.

\subsection{Linearity between Cerenkov measurements and dose}

The relationship of camera response to dose was examined by delivering different doses to the water phantom and imaging the emitted Cerenkov light. Figure 11 shows the dose linearity 

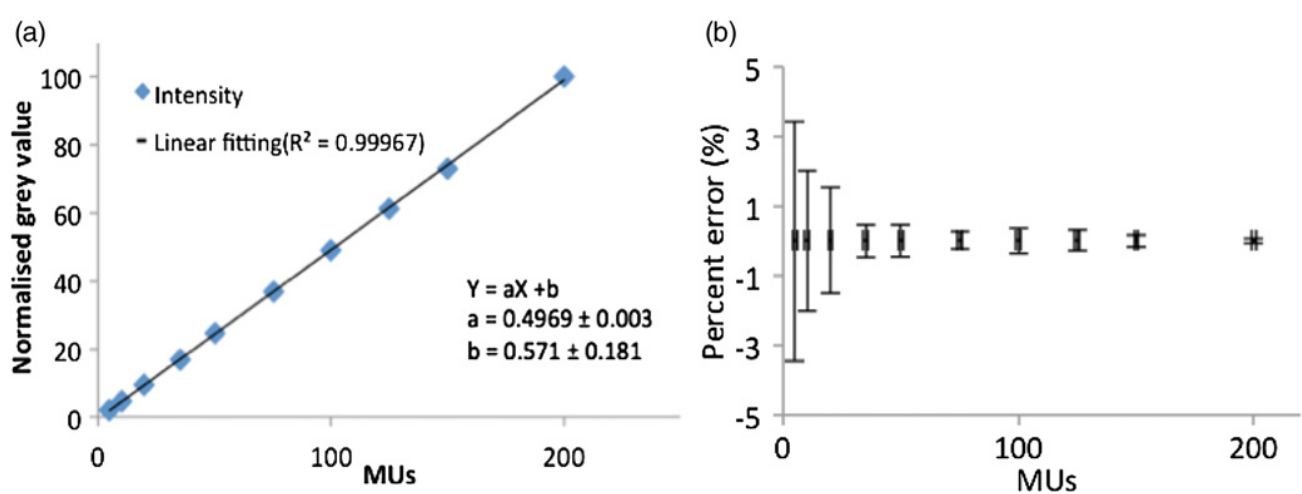

Figure 11. (a) Demonstration of dose linearity from 5 to $200 \mathrm{MU}$ with a $12 \mathrm{MeV}$ beam. All measurements were normalized to the $200 \mathrm{MU}$ measurement. (b) The percentage error of the measured data as a function of MUs. The error bars represent the standard deviation of four repeated readings.

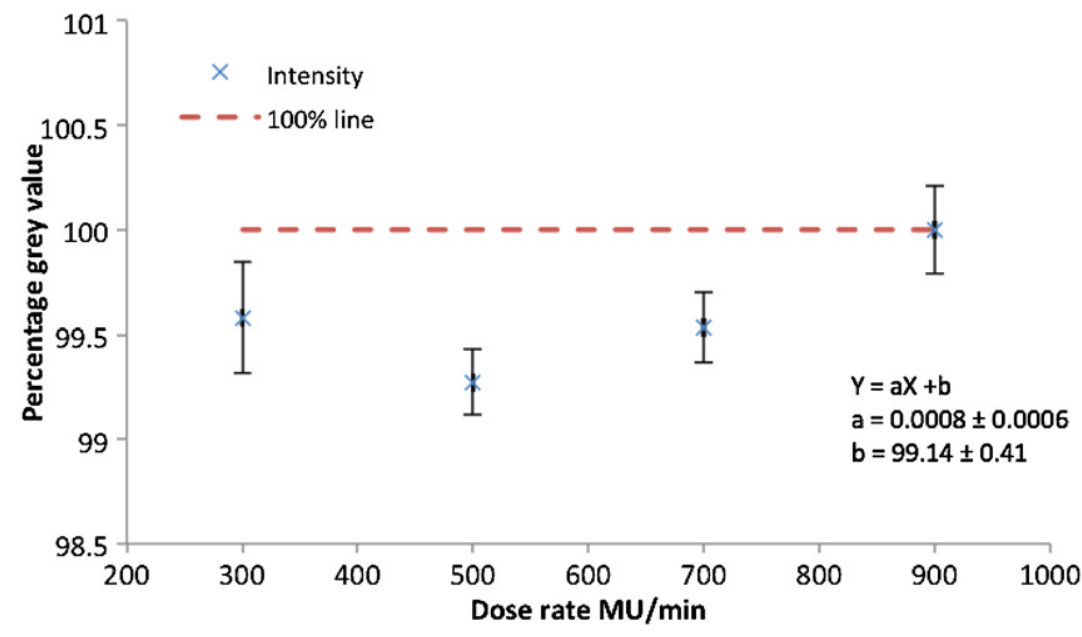

Figure 12. Dose rate measurements using Cerenkov-electron method for $12 \mathrm{MeV}$ electron beam. The variation is less than $0.65 \%$. All measurements normalized to

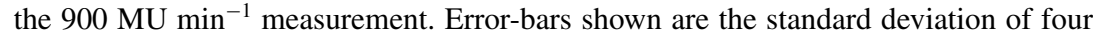
repeated reading.

of Cerenkov images using a $12 \mathrm{MeV}$ electron beam. All measurements are normalized to the 200 MU measurement.

It was found that the commercial digital camera was sensitive enough to detect the smallest dose delivered by our Linac ( $5 \mathrm{MU}$ ) within $3 \%$ uncertainty. A region of interest in the Cerenkov images equal to a square of $3 \times 3 \mathrm{~mm}^{2}$ which is the same size as a typical scanning ionization chamber was chosen to check the dose linearity. The goodness of the fitting to data $\left(R^{2}\right.$ value $)$ is better than 0.9997 . The percentage standard deviation in the worst case was $\pm 3.4 \%$ for the smallest MU delivered.

\subsection{Dependence of Cerenkov measurements on dose rate}

The linearity of Cerenkov emission detection with dose rate was measured by delivering different dose rates and imaging the emitted Cerenkov light. Figure 12 shows the dose rate 


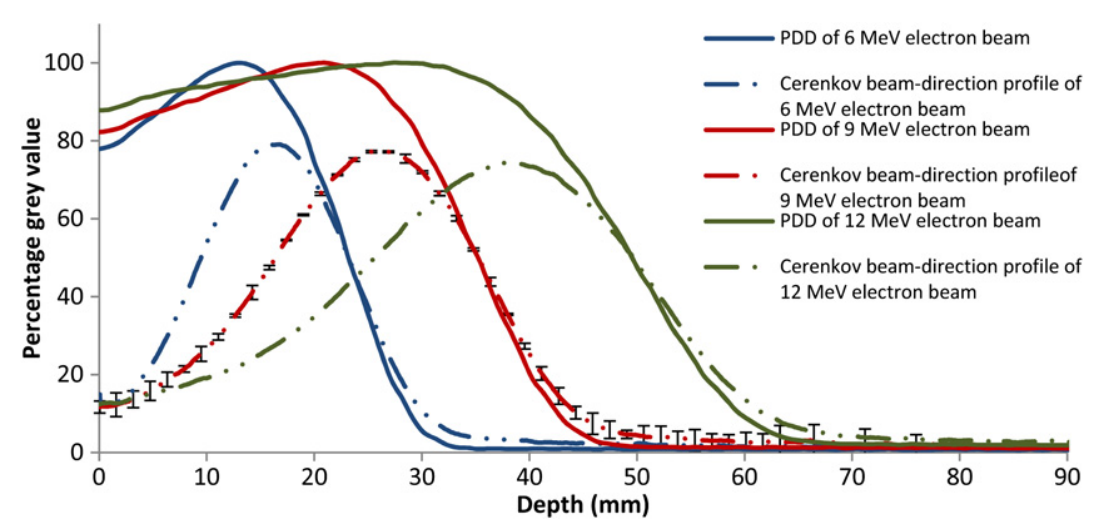

Figure 13. Beam-direction profiles for different electron energies compared with electron depth doses measured by ionization chamber. Error-bars shown are the standard deviation of four repeated reading of $12 \mathrm{MeV}$ measurements.

dependence in the Cerenkov images averaged over $3 \times 3 \mathrm{~mm}^{2}$ squares, using a $12 \mathrm{MeV}$ electron beam. The variation of the dose rate was found to be less than $0.65 \%$, compared to $0.6 \%$ with an ionization chamber due to the variation in accelerator output found by Li et al (2013). All measurements were normalized to the $900 \mathrm{MU} \mathrm{min}^{-1}$ measurement.

\subsection{Range measurements}

To measure the practical range of the electron beams in water, the measured beam-direction profiles for different electron energies were compared to depth dose profiles measured by the ionization chamber. Because the depths of dose maximum and of Cerenkov light maximum do not coincide (see section 3.1.4 above), all data were normalized to match at the depth of $50 \%$ of dose maximum, as shown in figure 13 . The practical electron ranges measured in the Cerenkov images and by the ionization chamber were calculated and compared as explained in section 2.6.

For range measurements, an average was taken over steps of $1 \mathrm{~mm}$ to satisfy the clinical requirement of $\leqslant 2 \mathrm{~mm}$. It can be seen from figure 13 that the slope of the descending portion of the Cherenkov beam-direction profile is gentler than that for the depth doses. This is due to magnification effect (as explained in section 2.9.1), and it results in an overestimate of the practical range derived from the Cherenkov images. The ranges were 29.5 and $30.5 \mathrm{~mm}$ for the PDD and Cerenkov measurements for the $6 \mathrm{MeV}$ beam, 43.5 and $45.0 \mathrm{~mm}$ for the $9 \mathrm{MeV}$ beam and 60.0 and $63.0 \mathrm{~mm}$ for the $12 \mathrm{MeV}$ beam. The differences in depth between PDDs and the corresponding Cerenkov profiles increase with beam energies. This is due to magnification effects as described in section 2.9.1.

\subsection{Field size}

To estimate the width of the beam profile at $d_{\max }$, the transverse profiles measured in the Cerenkov images were compared to the ionization chamber measurements at $d_{\max }$ for $12 \mathrm{MeV}$ electron beam and $6 \times 6 \mathrm{~cm}^{2}$ field size as shown in figure 14. All data were normalized to their maximum value.

For field size measurement, again an average was taken over a $1 \mathrm{~mm}$ step in the $z$-axis to satisfy the clinical requirement of $\leqslant 2 \mathrm{~mm}$. The penumbra of the transverse profile measurements appears much wider than for the ionization chamber measurements. This is 


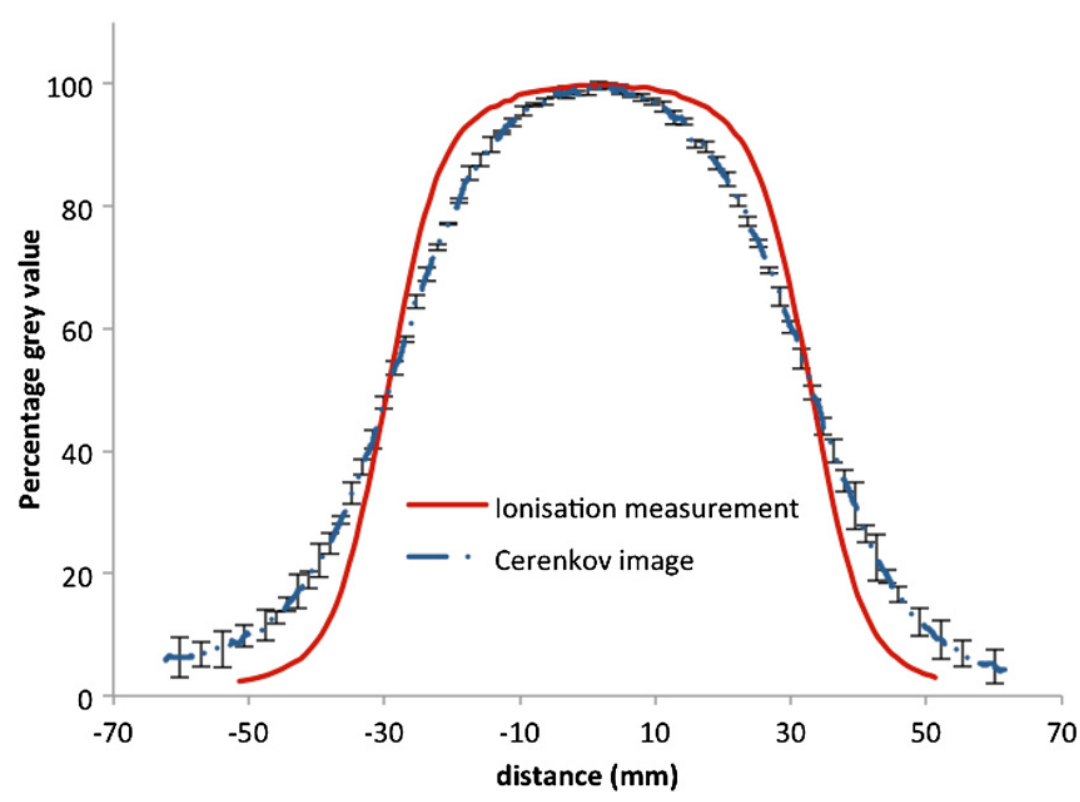

Figure 14. Transverse profiles at $d_{\max }$ for $6 \times 6 \mathrm{~cm}^{2}$ beam field measured by ionization chamber and Cerenkov images, The Cerenkov measurements were corrected for vignetting. Error-bars shown are the standard deviation of four repeated reading.

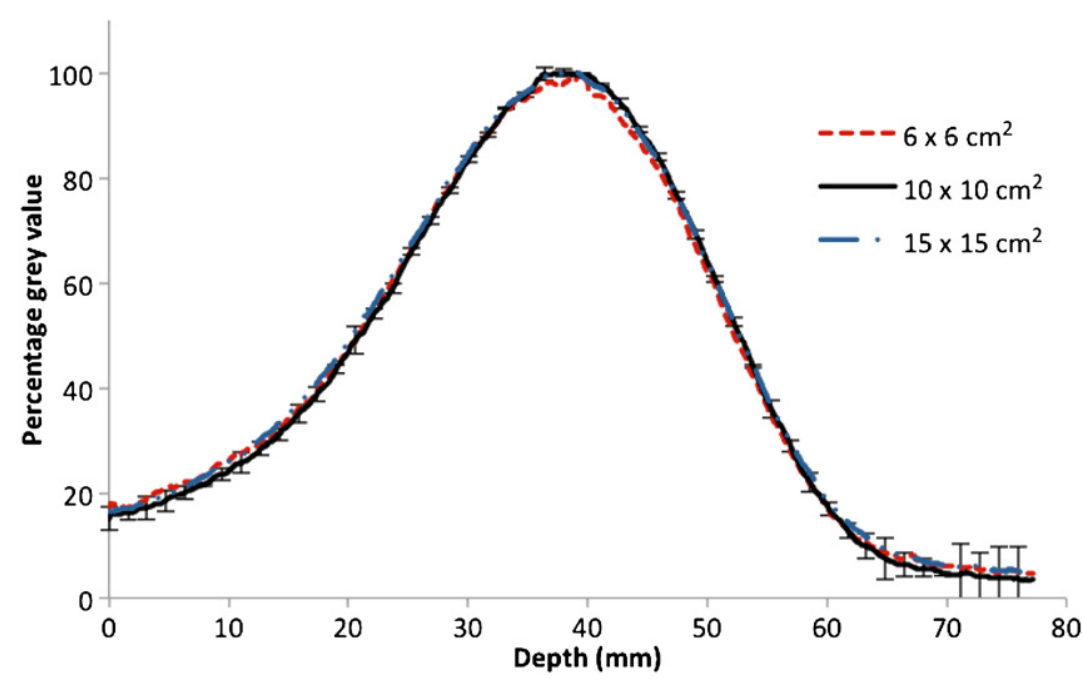

Figure 15. Beam-direction profiles for different collimator sizes, normalized to their respective maximum. Error-bars shown are the standard deviation of four repeated reading of $10 \times 10 \mathrm{~cm}^{2}$ measurements.

partly due to the magnification effect, as explained in section 2.9.1. This leads to the blurring effect which is apparent in figure 14. This could be solved by tomography from multiple camera images. However, noting that the blurring effect is symmetrical about the primary beam axis, the Cerenkov profile will provide an accurate measure of the beam width measured at 50\% of the maximum dose. For example, in figure 14, the beam profile at $50 \%$ of the maximum 
measured by the ionization chamber is $6.2 \mathrm{~cm}$ whereas measured by Cerenkov emissions it is $6.2 \pm 0.1 \mathrm{~cm}$.

\subsection{Field size dependence}

The dependence of the beam-direction profiles in Cerenkov images on the field sizes was explored. The measured beam-direction profiles for applicator sizes of $6 \times 6 \mathrm{~cm}^{2}$, $10 \times 10 \mathrm{~cm}^{2}$ and $15 \times 15 \mathrm{~cm}^{2}$ were compared for a $12 \mathrm{MeV}$ electron beam as shown in figure 15. All data were normalized to their maximum values. The beam-direction profile measurements generally matched to within $2 \%$ apart from the distal tail where the intensity was very low. Since depth dose profiles of electron beams are constant for large enough field sizes, the similarity of the Cerenkov beam-direction profiles shows that there is only a small influence from field size due to the magnification effect on the beam-direction profiles, as discussed in section 2.9.1. Therefore, this method can be used to check the constancy of electron range independently from the field size used.

\section{Conclusions}

A new potential quality assurance (QA) method for clinical electron beams was explored by measuring Cerenkov light from a therapeutic electron beam in a water tank. The light was imaged using a commercial camera which was sensitive enough to detect Cerenkov light in electron therapy.

A clinical electron beam in a water tank was modelled using a quasi-Gaussian electron energy spectrum and beam divergence and the resulting Cerenkov emissions were simulated. The implemented pinhole code was able to reproduce the experimental Cerenkov images taken at UCLH for different energies. By scoring the Cerenkov production profile along with the deposited energy, we were able to define the relation between them.

The short term repeatability of all measurements was found to be better than $1 \%$ except when measuring very low doses. Cerenkov light measurements were linear with dose and independent of dose rate.

Cerenkov beam-direction profiles were different from the depth dose profiles due to the factors mentioned in sections 3.1.3 and 2.9. By applying an in-house vignetting correction factor, the range could be retrieved with a maximum discrepancy of $3 \mathrm{~mm}$. Since the source of the discrepancy is a known geometrical effect, the differences between Cerenkov and depth dose ranges are expected to be constant for the same set-up, so this method can still be used to monitor any changes in beam energy. The beam-direction profiles were practically independent of field size.

Similarly, there was a difference between transverse profiles measured from Cerenkov images and the ionization chamber due to the magnification effects. However, Cerenkov profiles and ionization profiles meet at $50 \%$ level, so this method can still be used to monitor any changes in beam width.

In summary we found that imaging Cerenkov light during radiotherapy QA could be a suitable tool to measure dose and dose rate constancy with high precision. Beam-direction profiles and transverse profiles could be used as a very quick routine QA tool to check range and field width constancy of electron beams.

\section{Acknowledgment}

We would like to thank University College London Hospitals for their support and for letting us using their facility. 


\section{References}

Agostinelli S et al 2003 Geant4-a simulation toolkit Nucl. Instrum. Methods Phys. Res. A 506 250-303 Axelsson $\mathrm{J}$ et al 2011 Cerenkov emission induced by external beam radiation stimulates molecular fluorescence Med. Phys. 38 4127-32

Axelsson J et al 2012 Quantitative Cherenkov emission spectroscopy for tissue oxygenation assessment Opt. Express 205133

Boschi $\mathrm{F}$ et al 2011 In vivo ${ }^{18} \mathrm{~F}-\mathrm{FDG}$ tumour uptake measurements in small animals using Cerenkov radiation Eur. J. Nucl. Med. Mol. Imaging 38 120-7

Cleland M, Lisanti T and Galloway R 2004 Comparisons of Monte Carlo and ICRU electron energy versus range equations Radiat. Phys. Chem. 71 585-9

Geant4 User's Guide for Application Developers 2012 (Geneva: Geant4 Publications)

Glaser A K et al 2013 Projection imaging of photon beams by the Cerenkov effect Med. Phys. $\mathbf{4 0} 012101$

Hale G M 1973 Optical constants of water in the $200 \mathrm{~nm}$ to $200 \mathrm{um}$ wavelength region Appl. Opt. 12 555-63

$\mathrm{Hu} \mathrm{Z}$ et al 2010 Experimental Cerenkov luminescence tomography of the mouse model with SPECT imaging validation Opt. Express 1824441

$\mathrm{Hu} \mathrm{Z}$ et al 2012 Three-dimensional noninvasive monitoring iodine-131 uptake in the thyroid using a modified Cerenkov luminescence tomography approach PLOS ONE 7 e37623

Jelley J V 1958 Cerenkov Radiation and its Applications (Oxford: Pergamon)

Kasarova S N et al 2007 Analysis of the dispersion of optical plastic materials Opt. Mater. 29 1481-90

Knoll G F 1988 Radiation Detection and Measurement 2nd edn (New York: Wiley)

Li C, Mitchell G S and Cherry S R 2010 Cerenkov luminescence tomography for small-animal imaging Opt. Lett. 35 1109-11

Li G et al 2013 Evaluation of the ArcCHECK QA system for IMRT and VMAT verification Phys. Med. 29 295-303

Yuan L C L and Wu C-S 1961 Nuclear Physics (Methods of Experimental Physics vol 5)

Newman F et al 2008 Visual sensations during megavoltage radiotherapy to the orbit attributable to Cherenkov radiation Med. Phys. 3577

Podgorsak E B 2006 Radiation Physics for Medical Physicists (Berlin: Springer)

Ray S 2002 Applied Photographic Optics: Lenses and Optical Systems for Photography, Film, Video and Digital Imaging 3rd edn (Focal Press)

Robertson R et al 2009 Optical imaging of Cerenkov light generation from positron-emitting radiotracers Phys. Med. Biol. 54 N355-65

Smith S W 2003 Digital Signal Processing: A Practical Guide for Engineers and Scientists Newnes

Tearney G J et al 1995 Determination of the refractive index of highly scattering human tissue by optical coherence tomography Opt. Lett. 20 2258-60

Zhang R et al 2012 Cerenkov radiation emission and excited luminescence (CREL) sensitivity during external beam radiation therapy: Monte Carlo and tissue oxygenation phantom studies Biomed. Opt. Express 3 2381-94

Zhang R et al 2013 Superficial dosimetry imaging of Čerenkov emission in electron beam radiotherapy of phantoms Phys. Med. Biol. 58 5477-93 